\title{
Influência da taxa de juros e do preço da madeira em modelos de regulação florestal
}

\author{
Kaio Henrique Adame de Carvalho${ }^{1}$, Márcio Lopes da Silva ${ }^{1}$, Helio Garcia Leite ${ }^{1}$, Daniel Henrique Breda Binoti ${ }^{*}$, \\ Mayra Luiza Marques da Silva Binoti ${ }^{1}$
}

${ }^{1}$ Universidade Federal de Viçosa, Departamento de Engenharia Florestal, Cep 35760-000, Viçosa, MG, Brasil

“Autor correspondente:
danielhbbinoti@gmail.com

Termos para indexação:

Programação linear

Manejo florestal

Variáveis econômicas.

Index terms:

Linear programming

Forest management

Economic variables

Histórico do artigo:

Recebido em 24/06/2013

Aprovado em 19/05/2015

Publicado em 30/06/2015

doi: $10.4336 / 2015 . p f b .35 .82 .554$
Resumo - A regulação da produção florestal é complexa, devido ao grande número de variáveis relacionadas ou envolvidas no processo, sendo que as variáveis econômicas influenciam de forma consistente os resultados dos modelos de regulação. Sendo assim, este trabalho tem por objetivo analisar a influência da taxa de juros e do preço da madeira em alguns modelos de regulação florestal. Utilizou-se programação linear em um estudo de caso empregando dados de 10 povoamentos florestais, com a aplicação do software Regulação da Produção Florestal, que foi utilizado também para simular cenários de manejo e planejamento. Todos os povoamentos florestais foram regulados independentemente do método utilizado. Quanto maior o preço da madeira, maior a área de corte anual e menor a idade média de corte. A produção média anual não foi alterada, independente da taxa de juros, do preço da madeira, ou do método utilizado.

\section{Influence of interest rate and the wood price in forest regulation models}

\begin{abstract}
Forest production regulation is complex due to the large number of variables in the process. Economic variables consistently influence the results of regulation models. Therefore this study aims to analyze the influence of interest rate and wood price in some forest regulation models. Linear programming model was used in a studying case using data from 10 forest stands and the software Regulation of Forest Production, which was also used to simulate management and planning scenarios. All stands were regulated independently of the method used. It was observed that as the wood price raises, the annual cutting area becomes bigger and the average logging age decreases. The average annual production presented no change regardless of interest rate, wood price or the method used.
\end{abstract}

\section{Introdução}

O planejamento florestal nas últimas décadas tornou-se um tema complexo e diversificado onde vários fatores além daqueles relacionados à dinâmica da floresta têm assumido papel central, como as políticas governamentais, a opinião pública, as preocupações ambientais, entre outros. À medida que novas preocupações surgiram, sentiu-se a necessidade de modelar os sistemas florestais de forma integrada, incluindo aspectos ambientais, sociais, produtivos e econômicos (Silva, 2004; Bettinger, et al., 2009; Campos \& Leite, 2013), sendo fundamental para o gerenciamento das atividades florestais a aplicação de sistemas de apoio à tomada de decisão. No entanto, no Brasil o uso dos mesmos ainda é incipiente (Binoti, 2012).

Uma das maiores dificuldades encontradas no manejo florestal é a regulação. Uma floresta regulada é aquela em que todas as áreas da floresta estão uniformemente distribuídas em classes de idade iguais, 
tendo periodicamente a mesma produção volumétrica e qualidade técnica, onde o objetivo é assegurar uma produção continua e a sustentabilidade de diversos produtos e de usos da floresta (Rodrigues, 1997; Bettinger, et al., 2009).

As indústrias do setor florestal têm a necessidade de organizar extensas áreas de plantio bem como planeja-las. Ao longo do horizonte de planejamento o manejador tem que lidar com as variáveis que podem afetar o manejo das florestas. Dentre essas, destacam-se as variáveis econômicas, que mostram os desembolsos e lucros que as empresas terão ao longo do horizonte estudado, devendo ser, portanto, uma preocupação do manejador na utilização de modelos de regulação (Brun, 2002). O estudo dessas variáveis é importante, já que as mesmas influenciam diretamente o valor ótimo dos modelos de programação linear.

Os dois principais modelos utilizados para regulação da produção florestal são conhecidos como modelo I e modelo II (Johnson \& Scheurman, 1977). Segundo Rodriguez \& Moreira (1989), devido à sua maior simplicidade, o modelo tipo I tem sido mais intensamente difundido e utilizado no Brasil (Taube Netto, 1984; Rodrigues, 1997; Castro, 2007; Moreira, 2008; Binoti, 2010, dentre outros).

O modelo I de programação linear é, na maioria das vezes, formulado com uma função objetivo que maximiza o valor presente líquido global (VPL), que representa o lucro do projeto. Sendo assim, este trabalho tem por objetivo analisar a influência da taxa de juros e do preço da madeira em alguns modelos de regulação florestal.

\section{Material e métodos}

\section{Caracterização da área de estudo}

$\mathrm{O}$ estudo de caso foi conduzido empregando dados cadastrais de 10 povoamentos florestais localizados no estado de Minas Gerais. Esses povoamentos são constituídos de diversos clones de eucaliptos. Cada povoamento possui uma área total e distribuição de classes de idade distintas, porém todas divididas em 100 unidades de manejo (Tabela 1). A classe de idade atual de cada povoamento está representada na Tabela 2.

Tabela 1. Características dos povoamentos.

\begin{tabular}{cccc}
\hline Povoamentos & $\begin{array}{c}\text { Área total } \\
\text { (ha) }\end{array}$ & $\begin{array}{c}\text { Área média por } \\
\text { talhão (ha) }\end{array}$ & $\begin{array}{c}\text { Distância média dos } \\
\text { talhões à fábrica }(\mathrm{km})\end{array}$ \\
\hline 1 & 3020 & 30,20 & 22,72 \\
2 & 3108 & 31,08 & 26,34 \\
3 & 2989 & 29,89 & 27,01 \\
4 & 3005 & 30,05 & 22,97 \\
5 & 2875 & 28,75 & 25,39 \\
6 & 2899 & 28,99 & 26,05 \\
7 & 2958 & 29,58 & 25,04 \\
8 & 3010 & 30,10 & 25,42 \\
9 & 3219 & 32,19 & 24,38 \\
10 & 2848 & 28,48 & 25,70 \\
\hline
\end{tabular}

Tabela 2. Distribuição das áreas (ha) e das classes de idade inicial dos 10 povoamentos analisados.

\begin{tabular}{ccccccccccc}
\hline \multirow{2}{*}{ Idade (anos) } & \multicolumn{10}{c}{ Povoamentos } \\
\cline { 2 - 11 } & 1 & 2 & 3 & 4 & 5 & 6 & 7 & 8 & 9 & 10 \\
\hline 1 & 293 & 293 & 132 & 470 & 357 & 261 & 628 & 157 & 299 & 331 \\
2 & 148 & 307 & 440 & 135 & 90 & 320 & 485 & 318 & 371 & 304 \\
3 & 412 & 327 & 244 & 205 & 306 & 203 & 331 & 302 & 260 & 191 \\
4 & 411 & 252 & 347 & 259 & 436 & 227 & 315 & 114 & 305 & 244 \\
5 & 298 & 148 & 417 & 258 & 283 & 198 & 204 & 231 & 175 & 272 \\
6 & 323 & 378 & 141 & 348 & 212 & 308 & 277 & 111 & 490 & 224 \\
7 & 339 & 254 & 255 & 336 & 203 & 318 & 424 & 310 & 336 & 250 \\
8 & 286 & 520 & 322 & 401 & 363 & 287 & 294 & 378 & 320 & 394 \\
9 & 161 & 304 & 431 & 304 & 167 & 347 & 0 & 169 & 220 & 282 \\
10 & 349 & 325 & 260 & 289 & 458 & 430 & 0 & 131 & 443 & 356 \\
11 & 0 & 0 & 0 & 0 & 0 & 0 & 0 & 343 & 0 & 0 \\
12 & 0 & 0 & 0 & 0 & 0 & 0 & 0 & 163 & 0 & 0 \\
13 & 0 & 0 & 0 & 0 & 0 & 0 & 0 & 283 & 0 & 0 \\
\hline Total (ha) & 3.020 & 3.108 & 2.989 & 3.005 & 2.875 & 2.899 & 2.958 & 3.010 & 3.219 & 2.848 \\
\hline
\end{tabular}




\section{Formulação do problema:}

O modelo de programação linear foi formulado empregando a teoria do Modelo I, conforme descrito por Johnson \& Scheurman (1977). A solução foi obtida empregando o software RPF (Binoti, 2012), que foi utilizado também para simular cenários de manejo e planejamento.

Função Objetivo -1: Maximização do VPL

$$
\operatorname{Max} Z=\sum_{i=1}^{M} \sum_{j=1}^{N} V P L_{i j} X_{i j}
$$

Função Objetivo - 2: Maximização da produção

$$
V P L_{i j}=\sum_{k=1}^{H-1} R_{k}(1+r)^{-k}-C_{k}(1+r)^{-k}
$$

Ambas sujeitas a:

$$
\begin{aligned}
& \operatorname{Max} Z=\sum_{i=1}^{M} \sum_{j=1}^{N} P_{i j} X_{i j} \\
& \sum_{j=1}^{N} X_{i j} \leq A_{i} \forall i \quad(\mathrm{i}=1,2, \ldots, \mathrm{M})
\end{aligned}
$$

$$
\begin{aligned}
& \sum_{i=1}^{M} \sum_{j=1}^{N} V_{i j(k+1)} X_{i j} \geq D \min _{k} \\
& \sum_{i=1}^{M} \sum_{j=1}^{N} V_{i j(k+1)} X_{i j} \leq D \max _{k} \\
& \sum_{i=1}^{M} \sum_{j=1}^{N} X_{i j t}>\frac{S}{N C}-\left(\frac{S}{N C} * 0,05\right) \quad(\mathrm{t}=1,2, \ldots, \mathrm{NC})
\end{aligned}
$$

\section{Em que:}

$V P L_{i j}=$ valor presente líquido do fluxo de caixa de cada unidade de manejo i sobre a prescrição j, calculado ao longo do horizonte de planejamento, mais o valor presente do estoque final; $X_{i j}=$ área da unidade de manejo i assinalada à prescrição $\mathrm{j} ; \quad P_{i j}=$ Produção de cada unidade de manejo i sobre a prescrição j; $M=$ Número de unidades de manejo; $N=$ Número de prescrições $\mathrm{j}$ aplicada a cada unidade de manejo $\mathrm{i} ; R_{k}=$ Receita total no período k; $C_{k}=$ Custo total no período $\mathrm{k} ; r=$ Taxa de juros por período; $H=$ Tamanho do horizonte de planejamento; $A_{i}=$ área da unidade de manejo i; $V$ ${ }_{i j k}=$ Volume produzido pela unidade de manejo i sobre a prescrição j no período k; $D \min _{k}=$ demanda mínima no ano k; $D \max _{k}=$ demanda máxima no período k; $X_{i j k}=$ área da i-ésima parcela manejada sobre a j-ésima prescrição, onde as árvores terão t períodos no período final do horizonte de planejamento; $S=$ área total do povoamento; $N C=$ número de classes de idade.

Este modelo foi rodado com três taxas de juros e três preços de madeira diferentes (nove combinações para cada povoamento), sendo rodados 90 modelos diferentes de maximização do VPL. As taxas de juros utilizadas foram $8 \%, 10 \%$ e $12 \%$ ao ano e as receitas de 64,80 e $96 \mathrm{R} \$ \mathrm{~m}^{-3}$. As taxas de juros e preços de venda da madeira são embasados em valores praticados em financiamento e comércio local. A comparação foi realizada para analisar se a maximização pelo VPL é igual à maximização pela produção.

O horizonte de planejamento deve ser escolhido com base em vários fatores como, por exemplo, o objetivo do projeto, sendo que horizontes muito grandes possibilitam maiores erros de análise já que várias mudanças podem ocorrer durante o período, como: mudanças econômicas, alteração de custos, dentre outras. Por outro lado, horizontes curtos podem não levar em consideração acontecimentos importantes que podem ocorrer após o período adotado (Rezende \& Oliveira, 2008). No presente trabalho foi escolhido um horizonte de planejamento de 14 anos, para se avaliar de duas a três rotações, compatíveis com as opções de corte. As idades de corte utilizadas foram 4, 5, 6, 7 e 8 anos.

A idade de regulação utilizada foi de 6 anos. A máxima produção anual permitida foi de $200.000 \mathrm{~m}^{3}$ de madeira e a produção mínima anual de $150.000 \mathrm{~m}^{3}$, sendo estes valores compatíveis com a curva de produção e com a idade regulatória de 6 anos. Foi considerado que as operações de corte eram seguidas de imediata reforma.

\section{Estimativas de produção e custos}

As estimativas de produção futura, bem como os custos do projeto florestal e de colheita foram obtidos a partir do trabalho de Binoti (2010). A equação utilizada para se calcular a produção futura foi demostrada na equação 4. Os custos podem ser observados na Tabela 3 e equação 5 . O custo de colheita utilizado foi de $\mathrm{R} \$$ $20,00 \mathrm{~m}^{-3}$, sendo o custo de transporte de $0,5 \mathrm{R} \$ \mathrm{~km}^{-1}$ $\mathrm{m}^{-3}$ (Binoti, 2010).

$$
\text { Volume }\left(m^{3} \cdot h a^{-1}\right)=\frac{485,68}{1+7,4275 e^{-0,4662 l i d a d e(a n s)}}
$$


Tabela 3. Custos empregados para a avaliação econômica dos modelos de regulação florestal.

\begin{tabular}{|c|c|c|c|c|c|c|}
\hline Ano & Atividade & $\begin{array}{l}\text { Rendimento } \\
\text { (unid ha }^{-1} \text { ) }\end{array}$ & Unidade & $\begin{array}{c}\text { Custo } \\
\text { unitário }\end{array}$ & $\begin{array}{c}\text { Aplicação } \\
(\%)\end{array}$ & $\begin{array}{c}\text { Total } \\
\left(\mathrm{R} \$ \mathrm{ha}^{-1}\right)\end{array}$ \\
\hline $1^{\circ}$ ano & Adubação manual (calcário) & 20,00 & hh & 14,74 & $100 \%$ & 294,58 \\
\hline $1^{\circ}$ ano & Adubação NPK 06-10-29 & 13,00 & hh & 14,34 & $100 \%$ & 186,47 \\
\hline $1^{\circ}$ ano & Adubação NPK 06-30-06 & 10,00 & hh & 12,40 & $100 \%$ & 123,95 \\
\hline $1^{\circ}$ ano & Ajudante florestal & 20,00 & hh & 11,39 & $100 \%$ & 227,74 \\
\hline $1^{\circ}$ ano & Alinhamento/marcação & 10,00 & hh & 11,39 & $100 \%$ & 113,87 \\
\hline $1^{\circ}$ ano & Capina química manual total & 11,00 & hh & 14,51 & $80 \%$ & 127,68 \\
\hline $1^{\circ}$ ano & Capina química mecânica total & 0,95 & hh & 88,26 & $20 \%$ & 16,77 \\
\hline $1^{\circ}$ ano & Combate à formiga sistemático & 3,50 & hh & 11,93 & $100 \%$ & 41,75 \\
\hline $1^{\circ}$ ano & Combate à formiga Convencional & 8,00 & hh & 11,93 & $110 \%$ & 104,97 \\
\hline $1^{\circ}$ ano & Coveamento motorizado & 17,00 & hh & 22,99 & $80 \%$ & 312,63 \\
\hline $1^{\circ}$ ano & Irrigação caminhão pipa & 16,00 & hh & 18,57 & $100 \%$ & 297,18 \\
\hline $1^{\circ}$ ano & Limpeza manual & 80,00 & hh & 11,39 & $30 \%$ & 273,29 \\
\hline $1^{\circ}$ ano & Operador de motosserra & 2,00 & hh & 27,69 & $100 \%$ & 55,38 \\
\hline $1^{\circ}$ ano & Planificação & 5,00 & hh & 80,00 & $0 \%$ & ------ \\
\hline $1^{\circ}$ ano & Plantio com gel & 17,00 & hh & 16,71 & $100 \%$ & 284,00 \\
\hline $1^{\circ}$ ano & Replantio com gel & 7,00 & hh & 16,71 & $100 \%$ & 116,94 \\
\hline $1^{\circ}$ ano & Roçada manual & 30,00 & hh & 11,39 & $70 \%$ & 239,13 \\
\hline $1^{\circ}$ ano & Subsolagem/fosfatagem & 1,50 & hh & 135,70 & $20 \%$ & 40,71 \\
\hline $1^{\circ}$ ano & Calcário & 1,50 & $\mathrm{t}$ & 59,94 & $100 \%$ & 89,91 \\
\hline $1^{\circ}$ ano & Cupinicida & 0,03 & $\mathrm{Kg}$ & 845,13 & $100 \%$ & 27,89 \\
\hline $1^{\circ}$ ano & Formicida & 8,00 & $\mathrm{Kg}$ & 4,27 & $110 \%$ & 37,58 \\
\hline $1^{\circ}$ ano & Frete mudas/insumo & 1,00 & ha & 132,00 & $100 \%$ & 132,00 \\
\hline $1^{\circ}$ ano & Gel & 3,00 & $\mathrm{Kg}$ & 6,69 & $100 \%$ & 20,07 \\
\hline $1^{\circ}$ ano & Herbicida & 6,00 & $\mathrm{I}$ & 9,53 & $100 \%$ & 57,18 \\
\hline $1^{\circ}$ ano & Map & 0,33 & $\mathrm{Kg}$ & 1,35 & $100 \%$ & 0,45 \\
\hline $1^{\mathrm{o}}$ ano & Mudas & 1,20 & Mil & 231,40 & $100 \%$ & 277,71 \\
\hline $1^{\circ}$ ano & NPK 06-10-29 & 0,40 & $\mathrm{t}$ & 949,95 & $100 \%$ & 379,98 \\
\hline $1^{\circ}$ ano & NPK 06-30-06 & 12,00 & $\mathrm{t}$ & 939,71 & $100 \%$ & 113,57 \\
\hline $1^{\circ}$ ano & Topografia & 1,00 & ha & 65,67 & $100 \%$ & 65,67 \\
\hline \multicolumn{6}{|c|}{ Total ano 1} & 4059,05 \\
\hline $2^{\circ}$ ano & Adubação NPK 06-10-29 & 13,00 & hh & 14,34 & $100 \%$ & 186,47 \\
\hline $2^{\circ}$ ano & Ajudante florestal & 10,00 & hh & 11,14 & $100 \%$ & 111,40 \\
\hline $2^{\circ}$ ano & Capina química manual total & 15,00 & hh & 14,51 & $100 \%$ & 217,64 \\
\hline $2^{\circ}$ ano & Combate à formiga convencional & 6,00 & hh & 11,93 & $110 \%$ & 78,73 \\
\hline $2^{\circ}$ ano & Construção manual de aceiros & 200,00 & hh & 11,39 & $10 \%$ & 227,74 \\
\hline $2^{\circ}$ ano & Coroamento & 16,00 & hh & 11,39 & $100 \%$ & 182,19 \\
\hline $2^{\circ}$ ano & Roçada manual - pós-plantio & 16,00 & hh & 11,39 & $100 \%$ & 182,19 \\
\hline $2^{\circ}$ ano & Formicida & 7,00 & $\mathrm{Kg}$ & 4,27 & $110 \%$ & 32,88 \\
\hline $2^{\circ}$ ano & Herbicida & 3,00 & $\mathrm{I}$ & 9,53 & $100 \%$ & 28,59 \\
\hline $2^{\circ}$ ano & NPK 06-10-29 & 0,40 & $\mathrm{t}$ & 949,95 & $100 \%$ & 379,98 \\
\hline \multicolumn{6}{|c|}{ Total ano 2} & 1627,05 \\
\hline $3^{\circ}$ ano & Capina química manual total & 13,00 & hh & 14,51 & $100 \%$ & 188,62 \\
\hline $3^{\circ}$ ano & Combate à formiga convencional & 6,00 & hh & 11,93 & $110 \%$ & 78,73 \\
\hline $3^{\circ}$ ano & Manutenção manual de aceiro & 200,00 & hh & 11,39 & $10 \%$ & 227,74 \\
\hline $3^{\circ}$ ano & Roçada manual & 16,00 & hh & 11,39 & $100 \%$ & 182,19 \\
\hline $3^{\circ}$ ano & Formicida & 5,00 & $\mathrm{Kg}$ & 4,27 & $110 \%$ & 23,49 \\
\hline $3^{\circ}$ ano & Herbicida & 6,00 & $\mathrm{Kg}$ & 9,53 & $100 \%$ & 57,18 \\
\hline \multicolumn{6}{|c|}{ Total ano 3} & 757,95 \\
\hline $4^{\circ}$ ao $14^{\circ}$ ano & Capina química manual total & 6,00 & hh & 11,93 & $110 \%$ & 78,73 \\
\hline $4^{\circ}$ ao $14^{\circ}$ ano & Formicida & 2,00 & $\mathrm{Kg}$ & 4,27 & $110 \%$ & 9,39 \\
\hline \multicolumn{6}{|c|}{ Total ano 4 ao 14} & 88,12 \\
\hline
\end{tabular}




\section{Resultados e discussão}

Os modelos testados apresentaram soluções viáveis para todos os cenários avaliados, sendo que se implementou alterações nos modelos de regulação apenas nos povoamentos 7 e 8 , já que os mesmos não apresentaram solução viável. O povoamento 7 era muito jovem e não conseguiu suprir a demanda mínima de madeira para alguns anos, sendo que a estratégia utilizada para regulação foi diminuir a demanda de madeira do ano 4 para $100.000 \mathrm{~m}^{3}$. Outra possibilidade seria aumentar o número de alternativas para cada unidade de manejo. Já o povoamento 8 era mais velho, portanto a demanda de madeira nos anos iniciais extrapolava a demanda máxima. Neste caso, permitiu-se que as idades de corte variassem de 5 a 10 anos, sendo que assim obteve-se a regulação da produção florestal no prazo de 14 anos.

Em relação aos modelos analisados observa-se que o preço da madeira tem uma representatividade maior na variação do VPL quando comparado à taxa de juros (Tabela 4). Este resultado já era esperado visto que os custos representados pelo aumento da taxa de juros são bem menores proporcionalmente que a receita gerada pelo preço da madeira.

Tabela 4. Valor Presente Líquido global em milhões (VPL) para os 10 povoamentos em cada uma das diferentes modelagens, sendo "Pr" o preço da madeira.

\begin{tabular}{cccccccccccc}
\hline \multirow{2}{*}{ Povoamento } & \multicolumn{3}{c}{ Taxa de juros $8 \%$} & \multicolumn{3}{c}{ Taxa de juros $10 \%$} & \multicolumn{3}{c}{ Taxa de juros $12 \%$} \\
\cline { 2 - 10 } & \multicolumn{1}{c}{$\operatorname{Pr} 64$} & $\operatorname{Pr} 80$ & $\operatorname{Pr} 96$ & $\operatorname{Pr} 64$ & $\operatorname{Pr} 80$ & $\operatorname{Pr} 96$ & $\operatorname{Pr} 64$ & $\operatorname{Pr} 80$ & $\operatorname{Pr} 96$ \\
\hline 1 & 1,74 & 4,13 & 6,64 & 1,08 & 3,28 & 5,51 & 0,47 & 2,51 & 4,57 \\
2 & 1,81 & 4,28 & 6,79 & 1,1 & 3,38 & 5,69 & 0,44 & 2,55 & 4,68 \\
3 & 1,74 & 4,12 & 6,54 & 1,07 & 3,27 & 5,49 & 0,44 & 2,48 & 4,53 \\
4 & 1,74 & 4,14 & 6,55 & 1,07 & 3,27 & 5,5 & 0,43 & 2,48 & 4,54 \\
5 & 1,65 & 3,94 & 6,24 & 1,01 & 3,11 & 5,21 & 0,39 & 2,33 & 4,27 \\
6 & 1,69 & 4,03 & 6,39 & 1,02 & 3,17 & 5,34 & 0,37 & 2,37 & 4,36 \\
7 & 1,45 & 3,55 & 5,68 & 0,93 & 2,84 & 4,8 & 0,44 & 2,22 & 4,03 \\
8 & 1,66 & 4,16 & 6,67 & 0,87 & 3,11 & 5,42 & 0,36 & 2,1 & 4,22 \\
9 & 1,86 & 4,41 & 6,98 & 1,14 & 3,47 & 5,84 & 0,46 & 2,61 & 4,8 \\
10 & 1,63 & 3,88 & 6,14 & 0,99 & 3,05 & 5,11 & 0,38 & 2,27 & 4,17 \\
\hline
\end{tabular}

Todas essas variações podem ser observadas na Tabela 5. As maiores variações devido ao preço da madeira foram de aproximadamente $73,5 \%$ para a taxa de $8 \%$;
$80,5 \%$ para a taxa de $10 \%$; e de $90,5 \%$ para a taxa de $12 \%$. As variações devidas à taxa de juros foram em média de $75 \%$ para um preço da madeira de $64 \mathrm{R} \$ \mathrm{~m}^{-3}$; $40 \%$ para o preço de $80 \mathrm{R} \$ \mathrm{~m}^{-3}$; e de $30 \%$ para o preço de $96 \mathrm{R} \$ \mathrm{~m}^{-3}$.

Tabela 5. Variação do VPL em milhões quando se reduz o preço da madeira de $96\left(\mathrm{R} \$ \mathrm{~m}^{-3}\right)$ para $64\left(\mathrm{R} \$ \mathrm{~m}^{-3}\right)$ considerando cada taxa de juros (A); e quando se aumenta a taxa de juros de $8 \%$ para $12 \%$ considerando cada preço da madeira (B).

\begin{tabular}{ccccccc}
\hline \multirow{2}{*}{ Povoamento } & \multicolumn{3}{c}{ (A) - Taxa de Juros (\%) } & \multicolumn{3}{c}{ (B) - Preço da Madeira $\left(\mathrm{R} \$ \mathrm{~m}^{-3}\right)$} \\
\cline { 2 - 7 } & 8 & 10 & 12 & 64 & 80 & 96 \\
\hline 1 & 49,0 & 44,3 & 41,0 & 12,7 & 16,2 & 20,7 \\
2 & 49,8 & 45,9 & 42,4 & 13,7 & 17,3 & 21,1 \\
3 & 48,0 & 44,2 & 40,9 & 13,0 & 16,4 & 20,1 \\
4 & 48,1 & 44,3 & 41,1 & 13,1 & 16,6 & 20,1 \\
5 & 45,9 & 42,0 & 38,8 & 12,6 & 16,1 & 19,7 \\
6 & 47,0 & 43,2 & 39,9 & 13,2 & 16,6 & 20,3 \\
7 & 42,3 & 38,7 & 35,9 & 10,1 & 13,3 & 16,5 \\
8 & 50,1 & 45,5 & 41,6 & 16,0 & 20,6 & 24,5 \\
9 & 51,2 & 47,0 & 43,4 & 14,0 & 18,0 & 21,8 \\
10 & 45,1 & 41,2 & 37,9 & 12,5 & 16,1 & 19,7 \\
\hline
\end{tabular}

Quando observamos a área média de corte anual, notase que à medida que se aumenta a taxa de juros e o preço da madeira, a regulação pela maximização do VPL se aproxima da regulação pela maximização da produção, conforme observado na Tabela 6. Porém, nota-se que o preço da madeira influencia mais que a taxa de juros. A receita da madeira é diretamente proporcional à receita do projeto, desta maneira o aumento do preço da madeira contribuirá para o aumento da área de corte. Cabe ressaltar que quando se maximiza a produção se tem a máxima produção possível para a floresta respeitando-se as restrições do modelo.

Porém, observa-se que os povoamentos 5,6 e 10 praticamente não tiveram variação em suas áreas de corte média, onde o preço da madeira e a taxa de juros não influenciaram na busca da solução ótima. Isto possivelmente ocorreu em função das restrições que impedem a influência das variáveis testadas. Ou seja, as restrições de demanda, de área e de regulação não permitem variações das áreas de cortes anuais, ou essas variações são muito pequenas, não causando influência na solução ótima. Esses povoamentos apresentaram uma estrutura inicial que com as restrições consideradas, resultaram em modelos com apenas uma solução ótima. 
Tabela 6. Área de corte média anual (ha) para os 10 povoamentos.

\begin{tabular}{|c|c|c|c|c|c|c|c|c|c|c|}
\hline \multirow{2}{*}{ Método } & \multicolumn{10}{|c|}{ Povoamento } \\
\hline & 1 & 2 & 3 & 4 & 5 & 6 & 7 & 8 & 9 & 10 \\
\hline $\mathrm{P}$ & 507,1 & 519,5 & 496,9 & 499,1 & 452,8 & 464,3 & 481,2 & 489,1 & 534,5 & 440,1 \\
\hline CMP_8 & 421,7 & 428,0 & 416,3 & 419,3 & 412,6 & 410,9 & 451,7 & 420,6 & 434,2 & 413,1 \\
\hline CMP_10 & 429,6 & 445,3 & 425,3 & 424,3 & 412,4 & 410,8 & 447,8 & 431,2 & 454,0 & 411,4 \\
\hline CMP_12 & 438,1 & 459,3 & 425,7 & 430,2 & 412,4 & 412,5 & 447,8 & 439,4 & 478,0 & 412,0 \\
\hline VPL_8_64 & 463,0 & 475,6 & 459,6 & 460,9 & 443,9 & 447,5 & 450,6 & 456,3 & 489,5 & 440,0 \\
\hline VPL_8_80 & 477,7 & 489,4 & 478,1 & 481,9 & 450,6 & 461,9 & 452,8 & 468,0 & 504,8 & 440,1 \\
\hline VPL_8_96 & 501,3 & 504,2 & 491,2 & 493,3 & 452,8 & 463,0 & 479,2 & 475,1 & 526,3 & 440,1 \\
\hline VPL_10_64 & 466,8 & 477,0 & 461,4 & 463,8 & 450,4 & 451,8 & 450,6 & 444,4 & 491,0 & 440,0 \\
\hline VPL_10_80 & 491,4 & 503,7 & 489,1 & 483,5 & 452,8 & 463,0 & 477,1 & 474,3 & 525,9 & 440,1 \\
\hline VPL_10_96 & 503,2 & 511,2 & 494,3 & 495,7 & 452,8 & 463,0 & 479,2 & 488,3 & 527,5 & 440,1 \\
\hline VPL_12_64 & 473,8 & 473,1 & 470,6 & 472,9 & 450,6 & 457,5 & 450,6 & 442,9 & 478,7 & 440,0 \\
\hline VPL_12_80 & 501,1 & 511,1 & 492,1 & 492,9 & 452,8 & 463,0 & 479,2 & 463,8 & 526,3 & 440,1 \\
\hline VPL_12_96 & 505,7 & 516,0 & 496,8 & 498,6 & 452,8 & 463,0 & 479,2 & 488,1 & 528,3 & 440,1 \\
\hline
\end{tabular}

Na Tabela 7 observa-se a produção média anual. Não foi observada influência da taxa de juros e do preço da madeira. A única exceção foi o povoamento 8 , que apresentava uma estrutura inicial mais velha. Neste caso, observa-se que independente da modelagem, a produção não varia muito ao longo do horizonte de planejamento, pois as áreas mais velhas tendem a serem cortadas primeiro.

Tabela 7. Produção média anual de madeira $\left(1.000 \mathrm{~m}^{3}\right)$ para os 10 povoamentos.

\begin{tabular}{ccccccccccc}
\hline \multirow{2}{*}{ Método } & \multicolumn{10}{c}{ Povoamento } \\
\cline { 2 - 10 } & 1 & 2 & 3 & 4 & 5 & 6 & 7 & 8 & 9 & 10 \\
\hline P & 165,2 & 171,5 & 164,6 & 165,1 & 157,4 & 160,7 & 149,9 & 171,1 & 177,0 & 155,4 \\
CMP_8 & 152,6 & 156,1 & 151,8 & 152,0 & 150,0 & 150,0 & 146,4 & 156,8 & 160,7 & 150,0 \\
CMP_10 & 151,1 & 158,0 & 151,1 & 150,8 & 150,0 & 150,0 & 146,4 & 158,9 & 162,8 & 150,0 \\
CMP_12 & 152,3 & 159,2 & 151,1 & 151,9 & 150,0 & 150,0 & 146,4 & 159,2 & 164,6 & 150,0 \\
VPL_8_64 & 164,2 & 170,5 & 163,9 & 164,4 & 157,1 & 160,2 & 149,4 & 170,6 & 175,8 & 155,4 \\
VPL_8_80 & 164,9 & 171,1 & 164,6 & 165,2 & 157,3 & 160,6 & 149,6 & 171,1 & 176,4 & 155,4 \\
VPL_8_96 & 165,3 & 171,5 & 164,7 & 165,2 & 157,4 & 160,6 & 149,8 & 171,3 & 177,1 & 155,4 \\
VPL_10_64 & 164,4 & 170,6 & 164,0 & 164,5 & 157,4 & 160,4 & 149,4 & 162,5 & 175,9 & 155,4 \\
VPL_10_80 & 165,3 & 171,5 & 164,5 & 165,2 & 157,4 & 160,6 & 149,7 & 171,4 & 177,1 & 155,4 \\
VPL_10_96 & 165,3 & 171,4 & 164,7 & 165,2 & 157,4 & 160,6 & 149,8 & 171,1 & 177,1 & 155,4 \\
VPL_12_64 & 163,4 & 163,3 & 164,4 & 162,8 & 157,3 & 160,5 & 149,4 & 161,4 & 165,2 & 155,4 \\
VPL_12_80 & 165,0 & 171,4 & 164,4 & 165,0 & 157,4 & 160,6 & 149,8 & 165,3 & 177,1 & 155,4 \\
VPL_12_96 & 165,1 & 171,6 & 164,6 & 165,1 & 157,4 & 160,6 & 149,8 & 170,9 & 177,0 & 155,4 \\
\hline
\end{tabular}


A produtividade média e a idade média de corte foram influenciadas apenas pelo preço da madeira (Tabelas 8 e 9). Quanto menor o preço da madeira, maior a idade média de corte e menor a produtividade média. Esta maior produtividade pode ter efeitos negativos do ponto de vista técnico, pois atrasa o corte, podendo este ocorrer quando o incremento corrente anual (ICA) for decrescente. Quanto à idade, o mesmo é observado, pois quando se diminui o preço da madeira se atrasa a idade de corte, aumentando assim a idade média de corte.

Tabela 8. Idade média de corte (anos) para os 10 povoamentos.

\begin{tabular}{ccccccccccc}
\hline \multirow{2}{*}{ Método } & \multicolumn{7}{c}{ Povoamento } \\
\cline { 2 - 10 }$y$ & 1 & 2 & 3 & 4 & 5 & 6 & 7 & 8 & 9 & 10 \\
\hline P & 6,82 & 6,97 & 6,97 & 6,97 & 7,36 & 7,36 & 6,40 & 7,77 & 6,99 & 7,51 \\
CMP_8 & 7,80 & 7,89 & 7,93 & 7,88 & 7,90 & 7,95 & 6,71 & 8,41 & 8,01 & 7,88 \\
CMP_10 & 7,50 & 7,58 & 7,60 & 7,65 & 7,90 & 7,95 & 6,75 & 8,20 & 7,66 & 7,90 \\
CMP_12 & 7,36 & 7,35 & 7,59 & 7,54 & 7,90 & 7,90 & 6,75 & 8,04 & 7,27 & 7,88 \\
VPL_8_64 & 7,49 & 7,63 & 7,56 & 7,58 & 7,52 & 7,65 & 6,86 & 8,36 & 7,64 & 7,51 \\
VPL_8_80 & 7,26 & 7,41 & 7,27 & 7,24 & 7,40 & 7,40 & 6,82 & 8,15 & 7,41 & 7,51 \\
VPL_8_96 & 6,91 & 7,20 & 7,06 & 7,06 & 7,36 & 7,38 & 6,42 & 8,02 & 7,11 & 7,51 \\
VPL_10_64 & 7,43 & 7,61 & 7,53 & 7,53 & 7,41 & 7,58 & 6,86 & 8,07 & 7,62 & 7,51 \\
VPL_10_80 & 7,05 & 7,20 & 7,08 & 7,22 & 7,36 & 7,38 & 6,44 & 8,04 & 7,11 & 7,51 \\
VPL_10_96 & 6,88 & 7,09 & 7,01 & 7,02 & 7,36 & 7,38 & 6,42 & 7,78 & 7,09 & 7,51 \\
VPL_12_64 & 7,25 & 7,28 & 7,39 & 7,27 & 7,40 & 7,48 & 6,86 & 8,05 & 7,26 & 7,51 \\
VPL_12_80 & 6,89 & 7,09 & 7,03 & 7,05 & 7,36 & 7,38 & 6,42 & 7,86 & 7,11 & 7,51 \\
VPL_12_96 & 6,83 & 7,02 & 6,97 & 6,97 & 7,36 & 7,38 & 6,42 & 7,78 & 7,08 & 7,51 \\
\hline
\end{tabular}

Tabela 9. Produtividade média $\left(\mathrm{m}^{3} \cdot \mathrm{ha}^{-1}\right)$ dos 10 povoamentos analisados.

\begin{tabular}{ccccccccccc}
\hline \multirow{2}{*}{ Método } & \multicolumn{7}{c}{ Povoamento } \\
\cline { 2 - 10 }$y$ & 1 & 2 & 3 & 4 & 5 & 6 & 7 & 8 & 9 & 10 \\
\hline P & 325,7 & 330,1 & 331,4 & 330,8 & 347,6 & 346,1 & 311,5 & 349,8 & 331,1 & 353,1 \\
CMP_8 & 361,9 & 364,8 & 364,6 & 362,6 & 363,7 & 365,1 & 324,2 & 372,8 & 370,1 & 363,1 \\
CMP_10 & 351,6 & 354,7 & 355,2 & 355,4 & 363,7 & 365,1 & 327,0 & 368,5 & 358,7 & 364,6 \\
CMP_12 & 347,5 & 346,5 & 355,0 & 353,1 & 363,7 & 363,6 & 327,0 & 362,2 & 344,3 & 364,1 \\
VPL_8_64 & 354,6 & 358,4 & 356,6 & 356,6 & 354,0 & 358,1 & 331,7 & 373,9 & 359,2 & 353,2 \\
VPL_8_80 & 345,1 & 349,5 & 344,3 & 342,8 & 349,2 & 347,6 & 330,3 & 365,7 & 349,5 & 353,1 \\
VPL_8_96 & 329,8 & 340,3 & 335,4 & 335,0 & 347,6 & 346,9 & 312,6 & 360,6 & 336,5 & 353,1 \\
VPL_10_64 & 352,2 & 357,6 & 355,4 & 354,8 & 349,5 & 355,1 & 331,7 & 365,6 & 358,3 & 353,2 \\
VPL_10_80 & 336,3 & 340,5 & 336,4 & 341,7 & 347,6 & 346,9 & 313,7 & 361,3 & 336,7 & 353,1 \\
VPL_10_96 & 328,5 & 335,3 & 333,2 & 333,3 & 347,6 & 346,9 & 312,6 & 350,5 & 335,6 & 353,1 \\
VPL_12_64 & 344,8 & 345,2 & 349,3 & 344,4 & 349,2 & 351,0 & 331,7 & 364,4 & 345,2 & 353,2 \\
VPL_12_80 & 329,3 & 335,4 & 334,2 & 334,7 & 347,6 & 346,9 & 312,6 & 356,4 & 336,4 & 353,1 \\
VPL_12_96 & 326,4 & 332,5 & 331,4 & 331,1 & 347,6 & 346,9 & 312,6 & 350,2 & 335,1 & 353,1 \\
\hline
\end{tabular}


Quando se compara o método de maximização do VPL com a maximização da produção nota-se que à medida que se aumenta o preço da madeira e a taxa de juros a modelagem pela maximização do VPL tende a ser bem parecida com o método de maximização da produção, sendo que para alguns casos a modelagem foi igual.

Para os povoamentos 5, 6 e 10, a modelagem foi praticamente a mesma para todos os 10 modelos testados, isso possivelmente devido a suas distribuições iniciais juntamente com as restrições que não permitiram variações.

No manejo de recursos naturais temos como ferramenta a pesquisa operacional (PO), que ao longo dos anos se mostrou eficiente no tratamento de problemas envolvendo a alocação de recursos escassos. Devido à enorme complexidade e quantidade de variáveis envolvidas nos processos de tomada de decisão, diversas ferramentas da pesquisa operacional foram introduzidas na área florestal, possibilitando a redução de riscos e incertezas na tomada de decisão (Rodrigues, 2001, Binoti 2010). Dentre as ferramentas com aplicação no manejo dos recursos florestais estão: Programação Linear (PL), Programação Inteira (PI), Programação Não-linear (PNL), Programação Multiobjetivo (PMO) e Programação Dinâmica (PD) (Binoti, 2010).

A PL se destaca em especial nas aplicações de ordenamento e otimização da produção florestal (Castro, 2007; Binoti, 2010). A programação linear é considerada uma das ferramentas básicas da pesquisa operacional. Esta baseia-se na maximização ou minimização de uma função linear, chamada função objetivo, que representa o principal objetivo do gestor. Sendo esta função objetivo sujeita a várias equações ou inequações, chamadas de restrições (Leuschner, 1984).

Trabalho dessa natureza que avaliem de maneira ampla a influência de características econômicas nos modelos de regulação são poucos vistos que o processo de simulação desses modelos é algo trabalhoso, principalmente em função da carência de sistemas e ferramentas para esse fim.

Mello et al. (2005) avaliaram a influência de diversos cenários de regulação sobre o planejamento florestal e o controle de estoque de carbono. Os autores verificaram variações significativas na simulação de cenários em função das variações da taxa de juros.
Como as empresas florestais buscam a obtenção do maior retorno econômico possível de seus plantios, respeitando restrições ambientais e sociais, surge a necessidade de simulações de diversos cenários, visando redução de custos e aumento do retorno econômico.

A influência de fatores econômicos já é bem conhecida e estudada em analises de projetos florestais (Rezende \& Oliveira, 2008). Entretanto, para analise global de todos os plantios de uma mesma empresa de maneira simultânea simulações dessa natureza devem ser realizadas.

A forma de determinação dos preços de venda da madeira ou dos demais produtos advindos da floresta é crucial para a estratégia da empresa, e o estabelecimento de preços torna-se, às vezes, uma decisão para a alta administração, visto que, estes são extremamente irregulares e na maioria das vezes com grandes variações sazonais (Palda, 1976).

A determinação da correta taxa de desconto é uma atividade extremamente complexa, uma vez que ela varia de acordo com as características do projeto, da empresa, da conjuntura econômica, os riscos e as incertezas, a inflação, a duração do projeto ou horizonte de planejamento e a posição particular do investidor (Rezende \& Oliveira, 2008). Em função das grandes movimentações do setor florestal com relação a fundos de investimentos internacionais, essa atividade tornouse ainda mais complexa, visando uma maior exatidão e certeza na sua determinação.

Ferramentas gratuitas de geração de modelos de regulação como o sistema RPF possibilitam a implementação e a geração de modelos de regulação de maneira extremamente rápida e com demonstrações claras de todos os cálculos realizados. Tal ferramenta pode ser utilizada em aulas e trabalhos acadêmicos de maneira extremamente amigável.

\section{Conclusões}

A variação nos valores da taxa de juros e no preço da madeira influenciam a solução dos modelos de regulação da produção florestal.

Quanto maior o preço da madeira, maior a área de corte anual e menor a idade média de corte. A produção média anual não foi alterada, independente da taxa de juros, do preço da madeira, ou do método utilizado. 


\section{Referências}

BETTINGER, P.; BOSTON, K.; SIRY, L. P.; GREBNER, R. L. Forest management and planning. London: Academic, 2009. 331 p.

BINOTI, D. H. B. Estratégias de regulação de florestas equiâneas com vista ao manejo de paisagem. 2010. 145 f. Dissertação (Magister Scientiae) - Universidade Federal de Viçosa, Viçosa, MG.

BINOTI, D. H. B.; BINOTI, M. L. M. da S.; LEITE, H. G. RPF: regulação da produção florestal: versão 0,15. Viçosa, MG, 2011. Software.

BINOTI, D. H. B. Sistemas computacionais aplicados ao manejo florestal. 2012. 131 f. Tese (Doutorado em Ciência Florestal) Universidade Federal de Viçosa, Viçosa, MG.

BRUN, F. L. Influência do valor da madeira de mercado sobre o ordenamento de florestas plantadas para o suprimento parcial de uma indústria de celulose e papel: uma aplicação de programação linear. 2002. 160 f. Dissertação (Mestrado em Recursos Florestais) - Escola Superior de Agricultura "Luiz de Queiroz", Piracicaba.

CASTRO, R. R. Regulação de florestas eqüiâneas incluindo restrições de adjacência. 2007. 64 f. Dissertação (Magister Scientiae) - Universidade Federal de Viçosa, Viçosa, MG.

CAMPOS, J. C. C; LEITE, H. G. Mensuração Florestal: perguntas e respostas. 4. ed. Viçosa, MG: Ed da UFV, 2013. 605 p.

JOHNSON, K. N.; SCHEURMAN, H. L. Techniques for prescribing optimal timber harvest and investment under different objectives discussion and synthesis. Forest Science, Bethesda, v. 18, n. 1, p. 1-31, 1977.
LEUSCHNER, W. A. Introduction to forest resource management. New York: John Willey \& Sons, 1984. 298 p.

MELlO, A. A.; CARNIERI, C.; ARCE, J. E.; SANQUETTA, C. R. Planejamento florestal visando à maximização dos lucros e a manutenção do estoque de carbono. Cerne, Lavras, v. 11, n. 3, p. 205-217, 2005.

MOREIRA, J. M. M. A. P. A incorporação de corredores de conectividade em problemas de otimização para o planejamento de florestas industriais. 2008. 123 f. Tese (Doutorado em Ciências) - Escola Superior de Agricultura Luiz de Queiroz, Piracicaba.

PALDA, K. S. Determinação de preços e política mercadológica. São Paulo: Atlas, 1976. 135 p.

REZENDE, J. L. P. de; OLIVEIRA, A. D. de. Análise econômica e social de projetos florestais. 2. ed. Viçosa, MG: Ed da UFV, 2008. 486 p.

RODRIGUES, F. L. Regulação de florestas equiâneas utilizando programação linear. 1997. 117 f. Dissertação (Mestrado em Ciência Florestal) - Universidade Federal de Viçosa, Viçosa, MG.

RODRIGUEZ, L. C. E.; MOREIRA, R. M. Gerenciamento de florestas de Eucalyptus com modelos de programação linear. IPEF, Piracicaba, v. 6, n. 19, p. 1-15, 1989.

SILVA, R. T. Planejamento florestal, modelos de programação inteira multiobjectivos e aplicações. 2004. 120 f. Dissertação (Mestrado) - Faculdade de Economia, Coimbra.

TAUBE NETTO, M. Um modelo de programação linear para planejamento de florestas de eucalipto. Pesquisa Operacional, Campinas, v. 4, n. 1, p. 19-39, 1984. 
